\title{
Patrones de uso de las plantas medicinales en el Chocó y Cauca (Colombia)
}

\section{Patterns of use of medicinal plants in Chocó and Cauca (Colombia)}

\author{
Diana Carolina Valoyes Milán ${ }^{\mathrm{a}^{*}}$ \\ Leider Palacios Palacios ${ }^{\mathrm{b}^{*}}$
}

Fecha de Recepción: 03.02.2019

Fecha de Aceptación: 10.05.2020

Doi: https://doi.org/10.19053/01217488.v11.n2.2020.10583

\section{Resumen}

En el campo de la etnobotánica, en el Chocó biogeográfico, se han realizados muchas investigaciones que tienen como objeto los diferentes usos de las plantas. Muchas de estas investigaciones pretendían proporcionar productos comerciales a los gobiernos o empresas privadas, pero la mayoría de estas investigaciones se llevaron a cabo para compartir el conocimiento del uso de plantas medicinales a la población y para rescatar un conocimiento que está en riesgo de perderse. La importancia de estos trabajos nos permite profundizar en el conocimiento del uso y el manejo de especies vegetales en las distintas zonas origen de las mismas. Así se pueden ofrecer mejoras o alternativas de explotación. Por esta razón Se realizó una exhaustiva revisión bibliográfica sobre el uso de las plantas medicinales, usando sobre todo publicaciones relacionadas Cauca y Chocó

En total se obtuvieron 1178 registros sobre el uso de plantas medicinales; 902 para el departamento del Chocó y 276 para el departamento del Cauca. Útiles en el tratamiento de 130 enfermedades o dolencias diferentes, asociadas a 17 categorías medicinales producto de la revisión bibliográfica de 17 publicaciones. Se encontraron 766 especies de plantas vasculares de las que 392 sp. para Chocó y 374sp. para Cauca, agrupadas en 132 familias. Las familias más representativas en el Chocó están: Gesneriaceae (103 usos), Asteraceae (83), Piperaceae (65), Arecaceae (46) y Araceae (41). En el Cauca se destacarón: Piperaceae (132 usos), Asteraceae (127), Fabaceae (68) Arecaceae (66), Gesneriaceae (51). Los problemas de salud con mayor número de tratamientos en Chocó fueron mordeduras de serpiente (194 registros de uso), paludismo (100), fiebre (74), desordenes y trastornos del hígado (74) y dolor de cabeza (62), mientras que, en Cauca fueron mordedura de serpiente (209), heridas en la piel con (77), diarrea (58), fiebre (58) y desordenes y trastornos renales (41). Respecto a los grupos humanos, los Afrocolombianos obtuvieron el mayor número de registros, seguidos por los indígenas y los mestizos

Palabras clave: Plantas medicinales, patrones usos, Chocó, Cauca.

\section{Abstract}

In total, 1178 records were obtained on the use of medicinal plants; 902 for the department of Chocó and 276 for the department of Cauca. Useful in the treatment of 130 different diseases or ailments, associated with 17 medicinal categories product of the literature review of 17 publica- tions. 766 species of vascular

\footnotetext{
a Magíster en Biodiversidad, Universidad Autónoma de Madrid. Integrante del grupo de investigación Biosistemática , Universidad Tecnológica del Chocó "Diego Luis Córdoba".

"correo electrónico: dianavaloyes20@hotmail.com

b Magister en biología, Universidad Nacional de Colombia (Bogotá). Docente ocasional del programa de Biología. Líder del grupo de investigación Biosistemática Universidad Tecnológica del Chocó "Diego Luis Cordoba"

*correo electrónico: leipal@hotmail.com
} 
plants were found of which $392 \mathrm{sp}$. for Chocó and 374sp. for Cauca, grouped into 132 families. The most representative families in the Chocó are: Gesneriaceae (103 uses), Asteraceae (83), Piperaceae (65), Arecaceae (46) and Araceae (41). In Cauca, the following stand out: Piperaceae (132 uses), Asteraceae (127), Fabaceae (68) Arecaceae (66), Gesneriaceae (51). The health problems with the highest number of treatments in Chocó were snake bites (194 use records), malaria (100), fever (74), liver disorders and disorders (74) and headache (62), while, in Cauca were snake bite (209), skin wounds with (77), diarrhea (58), fever (58) and kid- ney disorders and disorders (41). Regarding human groups, Afro-Colombians obtained the high- est number of records, followed by indigenous and mestizos.

Keywords: Medicinal plants, uses patterns, Chocó, Cauca.

\section{INTRODUCCIÓN}

Las plantas medicinales constituyen un elemento primordial dentro de la cultura de los pueblos. Aunque no existen datos precisos para evaluar la extensión del uso global de plantas medicinales, la Organización Mundial de la Salud ha estimado que más del $80 \%$ de la población mundial utiliza la medicina tradicional para satisfacer sus necesidades de atención primaria de salud y que gran parte de los tratamientos tradicionales implica el uso de extractos de plantas o sus principios activos [1], [ 2], [3], [ 4].

En los últimos años han surgido diferentes organizaciones y científicos que apoya la integración de la medicina alopática con la medicina tradicional, por ello se han generado iniciativas para lograr la unificación los saberes empíricos con el conocimiento científico como una estrategia de reducir la carecía de los servicios de salud [ 5].

Tanto en Asia como en otros países, la medicina tradicional se usa como resultado de circunstancias históricas y creencias culturales, podemos comparar con el casos puntuales de África, aproximadamente el $80 \%$ de la población recurre a la medicina tradicional, en países industrializados como Australia el $82 \%$, en Canadá el $70 \%$, en Estados Unidos el 42\%, en Bélgica el 38\%, en España el $41 \%$, en Francia el 75\%, y en China, el $40 \%$ de la atención sanitaria está a cargo de la medicina tradicional [6].

En Latinoamérica las personas recurren a la medicina tradicional por problemas, como: envidia, susto, nervios, problemas músculo- esqueléticos o cuando experimentan fracasos en la medicina alopática, etc. En México la medicina tradicional está presente en un $14 \%$, Perú $47 \%$, Bolivia $71 \%$, Ecuador 38\%, Guatemala $66 \%$ y Chile el $8 \%$ [ 7].
En Colombia la medicina tradicional asociada al uso con plantas, desempeña un rol importante dada la multiculturalidad representada en la diversidad étnica y su alta biodiversidad por lo cual es reconocido. Culturalmente habitan comunidades afrocolombianas, mestizos y pueblos indígenas, algunos de ellos con lenguas propias quienes en su mayoría habitan en zonas de alta diversidad biológica como los departamentos del Chocó y Cauca donde enfermedades como el susto, es comprendida como una curación del espíritu más que de la parte física [8]

De acuerdo con la OMS: "una planta medicinal es definida como cualquier especie vegetal que contiene sustancias que pueden ser empleadas para propósitos terapéuticos o cuyos principios activos pueden servir de precursores para la síntesis de nuevos fármacos".

Según el último informe del Real Jardín Botánico de Kew sobre el estado de las plantas en el mundo, se estima que existen 369.000 especies de plantas vasculares de las cuales 31.128 tienen al menos un uso [9]. En este reporte, se comparan especies de plantas en 21 bases de datos y revelan que el mayor número de plantas con un uso documentado son aquellas que han sido usadas en la medicina [10]; [11].

Según la estimación botánica para el Chocó biogeográfico realizada por Rangel \& Rivera [12], se han registrado 4548 especies de plantas vasculares, incluidas en 270 familias y 1211 géneros. Todas estas especies están presentes en localidades de los departamentos de Chocó, Cauca, Antioquia, Nariño, Risaralda y Valle del Cauca de Colombia.

El último catálogo florístico para el Chocó biogeográfico colombiano incluye 6052 especies de plantas vasculares, $35 \%$ de las cuales son endémicas [13]. 
Los departamentos del Chocó y Cauca son áreas biodiversas, con un alto nivel de especies endémicas; esta zona incluye territorios cuyos ambientes naturales se encuentran entre los mejor conservados de América, por poseer extensas superficies de su bosque original relativamente libre de amenazas, lo que significa que en esos ecosistemas los procesos naturales biológicos y evolutivos continuarán generando y manteniendo la biodiversidad de que todos dependemos [14]. En este sentido, es válido resaltar que las regiones tropicales aportan dos tercios de las especies de plantas estimadas en la Tierra. En general, los bosques lluviosos tropicales son muy diverso y alberga un gran número de especies endémicas. Aproximadamente unos cuartos de todas las especies de plantas son endémicos de esta zona por lo cual está catalogada como un punto caliente de biodiversidad, Sin embargo, no existe suficiente información sobre la abundancia y distribución de las plantas medicinales en áreas tropicales y, menos aún, sobre los patrones de uso de las poblaciones nativas [15].

El uso curativo de plantas medicinales, como suplentes de las medicinas alopática, se utiliza desde que el hombre existe para curar o aliviar las enfermedades. Sin embargo, no existe todavía la suficiente evidencia científica que consolide a la medicina herbaria dentro de los sistemas de salud. El objetivo de la investigación es identificar patrones de uso de las plantas medicinales en el Chocó y Cauca (Colombia).

\section{METODOLOGÍA}

El estudio fue desarrollado en los departamentos de Chocó y Cauca (Colombia), el primero está situado geográficamente en el suroeste del país entre las regiones andina y pacífica; localizado entre los $00^{\circ} 58^{\prime} 54^{\prime \prime}$ y $03^{\circ} 19^{\prime} 04^{\prime \prime}$ de latitud norte y los $75^{\circ} 47^{\prime} 36^{\prime \prime}$ y $77^{\circ} 57^{\prime} 05^{\prime \prime}$ ' de longitud oeste. Cuenta con una superficie de

$29.308 \mathrm{~km} 2$ lo que representa el $2.56 \%$ del territorio nacional. Limita por el Norte con el departamento del Valle del Cauca, por el Este con los departamentos de Tolima, Huila y Caquetá por el Sur con Nariño y Putumayo y por el Oeste con el océano Pacífico; cuenta con 1’379.169 Habitantes. (Informe general de ciudades y departamentos 2019 ) y el segundo está situado en el occidente del país, en la región de la llanura del Pacífico; localizado entre los $04^{\circ} 00^{\prime} 50^{\prime \prime}$ y $08^{\circ} 41^{\prime} 32^{\prime \prime}$ de latitud norte y los $76^{\circ} 02$ ' $57^{\prime \prime}$ y $77^{\circ} 53$ ' $38^{\prime \prime}$ " de longitud oeste ${ }^{\circ} \mathrm{C}$ Limita por el Norte con la República de Panamá y el mar Caribe, por el Este con los departamentos de Antioquia, Risaralda y Valle del Cauca, por el Sur con el departamento del Valle de Cauca, y por el Oeste con el océano Pacífico (Informe del departamento del Chocó 2015). ambos se ubican a lo largo de la costa pacífica de Colombia donde su población; La población afrocolombiana supera los cuatro millones 262 mil personas que constituye el 10,5 por ciento de los habitantes del país, mientras que los mestizos censados superaron los cuatro mil 830. En 1983 en el país habitaban 532 mil 233 indígenas. Según el DANAE [16] en Colombia sobreviven 87 pueblos indígenas. Algunas personas continúan identificándose con etnias ya extinguidas como los Tayronas, Quimbayas, Calima, Yarinquíe4s, Chitareros y Panches.

\subsection{Revisión bibliográfica}

La caracterización de los patrones de uso de las plantas medicinales para los departamentos (Chocó y Nariño), se basó en la revisión exhaustiva de publicaciones científicas (año) con enfoque de biogeografía histórica sobre el aprovechamiento de las plantas medicinales. Para la selección de publicaciones se tuvo en cuenta referencias basadas en datos originales obtenidos de trabajo de campo, incluidos artículos científicos, libros, monografías, tesis de grado y postgrado (máster y doctorado). En la búsqueda de contenido y bibliografía científico- académica, se utilizaron fuentes como; Google Académico, Web of Science, SciELO, Radalyc, Biblioteca de la Universidad tecnológica del Chocó.

\subsection{Organización de los datos}

De cada publicación, se obtuvo la siguiente información (cuando estaba disponible): Descripción del uso, familia, género, nombre científico de la especie tal como fue publi- cada, departamento, asignación a categorías de uso medicinal (ver en análisis de datos), si era cultivada o silvestre, y 
grupo étnico. Además, para cada uso medicinal se anotó la parte de la planta que utilizan, el nombre vulgar, la preparación y la forma de administración para el tratamiento de cada enfermedad.

Se reconocieron tres grupos étnico según el departamento al que pertenecieran: Afrocolombianos indígenas y mestizos. Dentro de los indígenas se registraron cinco grupos étnicos Awà, Cuna, Emberá, Waunana y Zenù.

Todos los usos medicinales registrados de la literatura fueron clasificados en 19 categorías medicinales siguiendo la Economic Botany Data Collection Standard [17], con algunas modificaciones propuestas por Macía et al. [18] para adaptarlo a áreas tropicales. Se incluyeron también las categorías (Síntomas sin especificación alguna y otros) propuestas por Gruca et al [19]. Cuando una descripción de uso medicinal no tuvo información suficiente para asignarla a una de las subcategorías descritas, se incluyó en la subcategoría "Sin especificación alguna".

\subsection{Actualización de la nomenclatura}

La actualización de la nomenclatura se realizó en base al reciente catálogo de plantas y líquenes de Colombia (Bernal et al., 2016), además se consultaron las páginas web: de http://www. ipni.org (Índice internacional de nomenclatura de plantas), http://www.tropicos.org (Misuri Botanical Garden) y http://www.theplantlist.org (The Plant list).

\subsection{Análisis de datos}

El análisis de datos se realizó a nivel de familia, género y especie. se tuvieron en cuenta los usos medicinales de las plantas en Chocó y Nariño en diferentes grupos humanos (Afrocolombianos, indígenas y mestizo). Consideramos como uso medicinal como aquel que refiere a una parte de la planta asociándolo con una categoría médica y una dolencia o enfermedad específica. Para la abundancia de usos dentro de las plantas medicinales, utilizando el término "registro de uso medicinal"'[20].

Por último, obtuvimos el porcentaje de especies útiles y de las diferentes categorías medicinales en función de la abundancia de cada una de las familias y sus usos en los diferentes departamentos para los distintos grupos humanos. Además, se comparó la presencia o no de cada una de las especies en los diferentes departamentos y relacionamos a cada grupo étnico con cada una de las variables propuestas (enfermedades, categoría medicinal, usos medicinales y parte de la planta usada) mediante la realización de tablas dinámicas con Excel.

\section{RESULTADOS Y DISCUSIÓN}

\subsection{Uso de plantas medicinales por departamento}

En total se obtuvieron 1178 registros de uso de plantas medicinales; 902 para el departamento del Chocó y 276 para el departamento del Cauca. Útiles para el tratamiento de 130 enfermedades o dolencias diferentes, asociadas a 17 categorías medicinales producto de la revisión bibliográfica de 17 publicaciones. El promedio de especies medicinales por publicación fue de $77 \pm 114$. Se registró información medicinal de cuatro grupos indígenas para el departamento del Chocó y uno en el departamento del Cauca, de mestizos se registraron solo para el departamento de Cauca y Afrocolombianos se registraron ambos departamentos (Tabla 1)

Tabla 1. Comparación por departamentos vs. variables de estudio

\begin{tabular}{|c|c|c|}
\hline Variables & Chocó & Cauca \\
\hline $\mathrm{N}^{\circ}$ de registro científicos & 902 & 276 \\
\hline Categoría medicinal & 17 & 16 \\
\hline Enfermedades & 118 & 38 \\
\hline Grupo étnico & 2 & 3 \\
\hline
\end{tabular}

El 65\% de las especies medicinales son plantas nativas y el 15\% de ellas son endémicas, mientras que el $11 \%$ son introducidas. El 16\% del total de las especies son cultivadas, también se obtuvo información que la mayoría de las plantas medicinales reportadas para los departamentos de Chocó y Cauca son palmas, hierbas y arbustos, las cinco familia que obtuvieron un mayor número de especies de plantas medicinales fueron: Piperaceae, Asteraceae, Arecaceae, Fabaceae, y malvaceae, Rubiaceae con el mismo valor de especies medicinales (Figura 1). 
Las partes de las plantas más utilizadas son las hojas (41\%), la planta entera (17\%) y la raíz (15\%) (Tabla 2). Cabe mencionar que para el 5\% de los registros no existe información sobre la parte de la planta.

Tabla 2. Partes de las plantas utilizadas con fines medicinales en el departamento de Chocó y Cauca.

\begin{tabular}{l|c}
\hline $\begin{array}{c}\text { Parte de la } \\
\text { planta }\end{array}$ & $\begin{array}{c}\text { Número de } \\
\text { reportes }\end{array}$ \\
\hline Hoja & 515 \\
\hline Planta entera & 215 \\
\hline Raíz & 185 \\
\hline Fruto & 100 \\
\hline Tallo & 52 \\
\hline Corteza & 32 \\
\hline Semillas & 28 \\
\hline Ramas & 22 \\
\hline Flores & 10 \\
\hline Bejuco & 27 \\
\hline Espinas & 27 \\
\hline Látex & 24 \\
\hline Bulbo & 29 \\
\hline
\end{tabular}

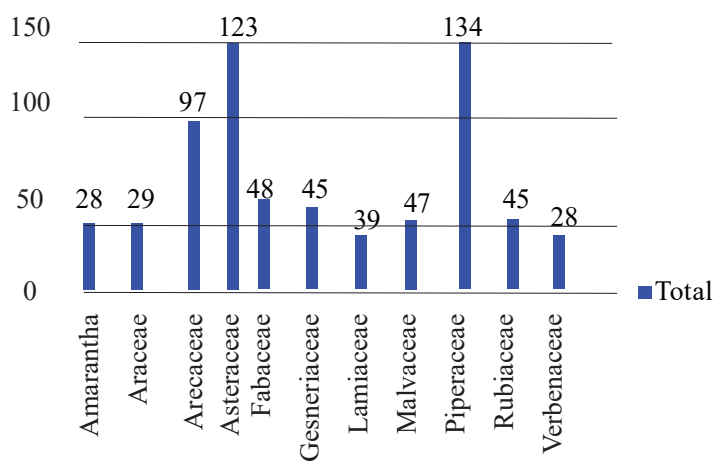

Figura 1. Familias de plantas con mayor numero especies medicinales en el Chocó y Ecuador

Las especies más importantes de acuerdo al número de usos medicinales; en la figura 2 podemos observar las comparaciones entre ambos departamentos (Chocó-Cauca). Para el departamento del Chocó las especies más significativas fueron: Coco nucifera (Coco), Manicaria saccifera las cuales pertenecientes a la familia de las Arecaceae (Palmas), Peperomia pellucida (Celedonia), Piper tricuspe (Kana) familia Piperaceae y Pouteria sapota (Zapote) familia Sapotaceae y en el departamento del
Cauca las especies destacadas fueron: Peperomia pellucida (Celedonia) perteneciente a la familia de las Piperaceae Alternanthera lanceolata(Discansel) familia Amaranthaceae, Trichanthera gigantea (Nacedera) familia acantáceas, Phytolaccas riverenoides (Jaboncillo) familia Phytolaccaceae, Hamelia sanguínea (merey) de la familia Rubiaceae.

En el caso del departamento del Chocó las dos especies de mayor importancia de acuerdo al número de usos medicines, pertenecen a la familia de las Arecaceae. Las palmas son uno de los grupos más utilizados y promisorios entre los bosques del pacífico colombiano además esta zona aloja la mayor diversidad y riqueza de palmas de todo el Neotrópico [21]. Por tal razón son asequibles a los pobladores de estas regiones lo que las posiciona como las más utilizadas para satisfacer necesidades básicas (medicina, casa, alimento etc.). Todas las partes de ella puede ser utilizada en materia medical; sus frutos, sus hojas, flores, tallo, raíces y aceites derivado de la mismas proporciona la cura para muchas enfermedades [22].

Estudio recientes como el de Escalante y Martínez [23]; revelan la importancia de la especie que reporta el mayor número de usos medicinales en el departamento del Chocó; Cocos nutifera (Coco), él demuestra como el aceite de esta planta puede actuar como actividad antibacteriana resolviendo un problema de salud preocupante, la resistencia de los antibióticos a las bacterias. Dejando en evidencia que los patrones de usos medicinales establecidos empíreamente por los habitantes de los pueblos de las zonas tropicales cada vez más adquieren rigor científico.

Otra de las palmas representativas fue Manicaria saccifera(Taparo) una especie de importancia a nivel financiero y con un gran número de comunidades silvestres en la región del pacífico, la cual reporta un gran número de usos [21]. Es de resaltar que es más conocida por que su bráctea peduncular es utilizada para realizar artesanías (sombreros de cabecinegro) pero reciénteme en un estudio sobre Morichales, Cananguchales (Moriche)y otros palmares inundables de suramérica se reportó su uso medicinal por parte de los indígenas Wounaan, Emberas y Tules, y de la comunidad afrocolombiana; los cuales afirman que, el endospermo líquido de la semilla puede ser utilizado para el tratamiento de los riñones, siendo igualmente comercializado en las calles Quibdó y la capital de Colombia- Bogotá[24]. 


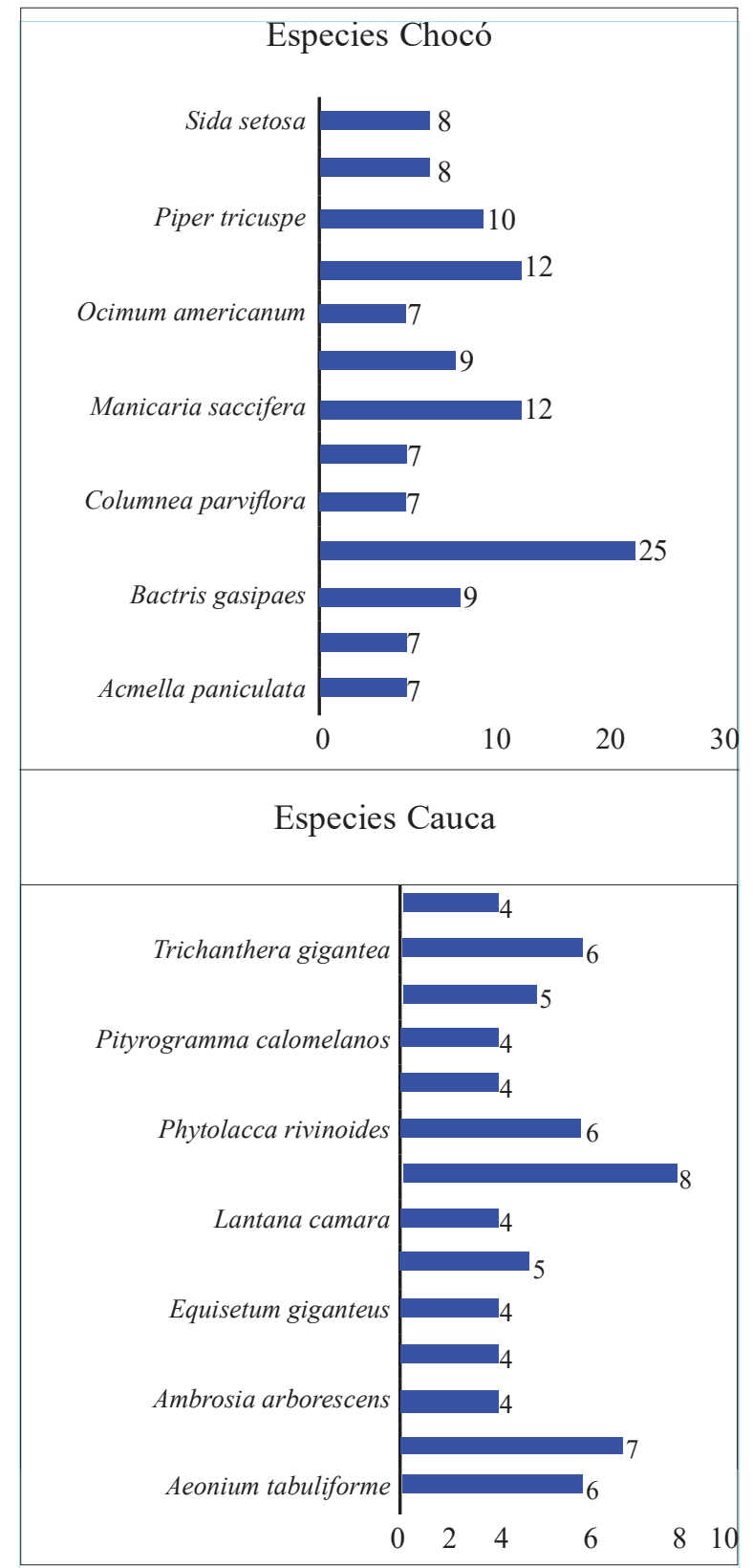

Figura 2. Especies más importantes de acuerdo al número de usos medicinales

La familia Piperaceae arrojo un importante número de usos en ambos departamentos (ChocóCauca). Las especies de este grupo, son planta de perfil amplio en cuanto a la aplicación para problemas de salud: prevención, diagnósticos y procesos terapéuticos o curas de enfermedades [25].

En el pacífico colombiano las especies de género Piper son utilizada como medicina, especialmente para deshinchar (como coloquialmente dicen las comunidades negra e indígenas que habitan esta región) o antibiótico, nombrándola como cordoncillo o deshinchadoras [26] también han sido empleadas por estos mismos pobladores para el tratamiento de enfermedades tropicales como la malaria [27].

Nuestros resultados revelan que Peperomia pellucida (Celedonia) es utilizada frecuentemente para tratar problemas diuréticos con un modo de administración frecuente de infusiones. Lo cual concuerda por lo dicho por Veloz y Martínez [28] en un estudio sobre la validación de efectos terapéutico de esta misma especie; ellos también afirman en sus conclusiones que esta especie es utilizada para enfermedades culturales conocidas como el mal de orina, es decir que la ingesta de esta planta regula problemas urinarios por tal motivo es mencionada como diurética. En este mismo sentido Piper tricuspe (Kana) fue registrada en nuestro estudio para la cura de enfermedades infecciosas y para el paludismo. Siendo reportada también por Benítez y Stashenko [29] como una especie útil para el tratamiento de enfermedades infecciosas.

Alternanthera lanceolata conocida en el argot étnico de los pueblos del andén pacífico colombiano como discansel grande o simplemente discansel. Reflejo usos significativos en el departamento del Cauca como la especie con mayor registro, la cual es suministrada vía oral como relajante y antiinflamatorio (nervios, dolor de cabeza, preocupación, etc.) y para casos de insolación. Una planta importante en la industria farmacéutica por poseer compuestos como fenoles, asteroides, flavonoides y alcaloides [30].

También se encontraron tratamiento interesante para la cura de la malaria. Schinz. Mesa et al., hallaron que los extractos del tallo de esta especie contienen una actividad antimalárica contra Plasmodium falciparum [31]. De forma similar, Quintanilla et al. [32] en estudio reciente de caracterización los compuestos fenólicos presentes en el extracto etanólico de hojas de Alternanthera lanceolata donde se demuestra claramente sus propiedades medicinales.

Trichanthera gigantea una especie muy popular en ambos departamentos conocida por las comunidades étnicas de la zona como nacedera, es utilizada por las parteras en niños recién nacidos para limpiar restos de placenta. Ríos [33] afirma que en la costa pacífica colombiana se dice que el nacedero es el árbol de la mujer y es utilizado en formas diversas antes y después del parto. También 
tiene otros múltiples usos en materia medicinal como los mencionado por Suárez y Milera [34] y para tratar trastornos respiratorios y digestivos, afecciones del hígado y los riñones y lesiones en la piel, como antihipertensivo y antipirético, para curar hernias y reducir el peso corporal, así como en los sistemas endocrino y muscular.

Hamelia sanguínea especie muy importante en el Cauca, utilizada para enfermedades como azúcar en la sangre, resultados que son confirmados en un estudio realizado por Martínez et al. [35]en donde se encontró que la administración de dosis repetidas de extracto de esta especie, ocasionaron una disminución significativa de glucosa en sangre comparable con el efecto de la metformina. El análisis bioquímico muestra que el extracto también ocasiona una disminución de triglicéridos.

En este mismo sentido, la investigación arrojo especies con mayor número de usos para el departamento del Chocó (Figura 3). Este alto porcentaje lo atribuimos a que este departamento posee gran biodiversidad, su recurso florístico se ha estimado entre 7,000 y 8,000 especies, y muchas de estas especies son endémicas [36]. En ese sentido, su flora puede ser comparada con la de una región con mega diversidad, que se encuentra altamente amenazada, y sin conocimiento de gran parte de ella. El registro Biogeográfico de la flora del Chocó, incluye 4,525 especies de espermatofitos con 170 familias distribuidas en 167 angiospermas y 3 gimnospermas [37]. Por tal motivo, esta zona es objeto de muchos estudios en etnobotánica, contrario a lo que se denota en el departamento del Cauca con la carecía de este tipo de indagaciones etnobotánicas. También es un hecho evidente que en el transcurrir de los años, las costumbres en el departamento del Chocó, han estado marcadas con la utilización de especies vegetales, a las cuales, empíricamente se les han atribuido facultades curativas para un sinnúmero de enfermedades, tradición que ha sido transmitida por generaciones hasta nuestros días. Por el lado del departamento del Cauca es un departamento que se encuentra más influenciada por la medicina alopátrica y con un porcentaje menor de biodiversidad.

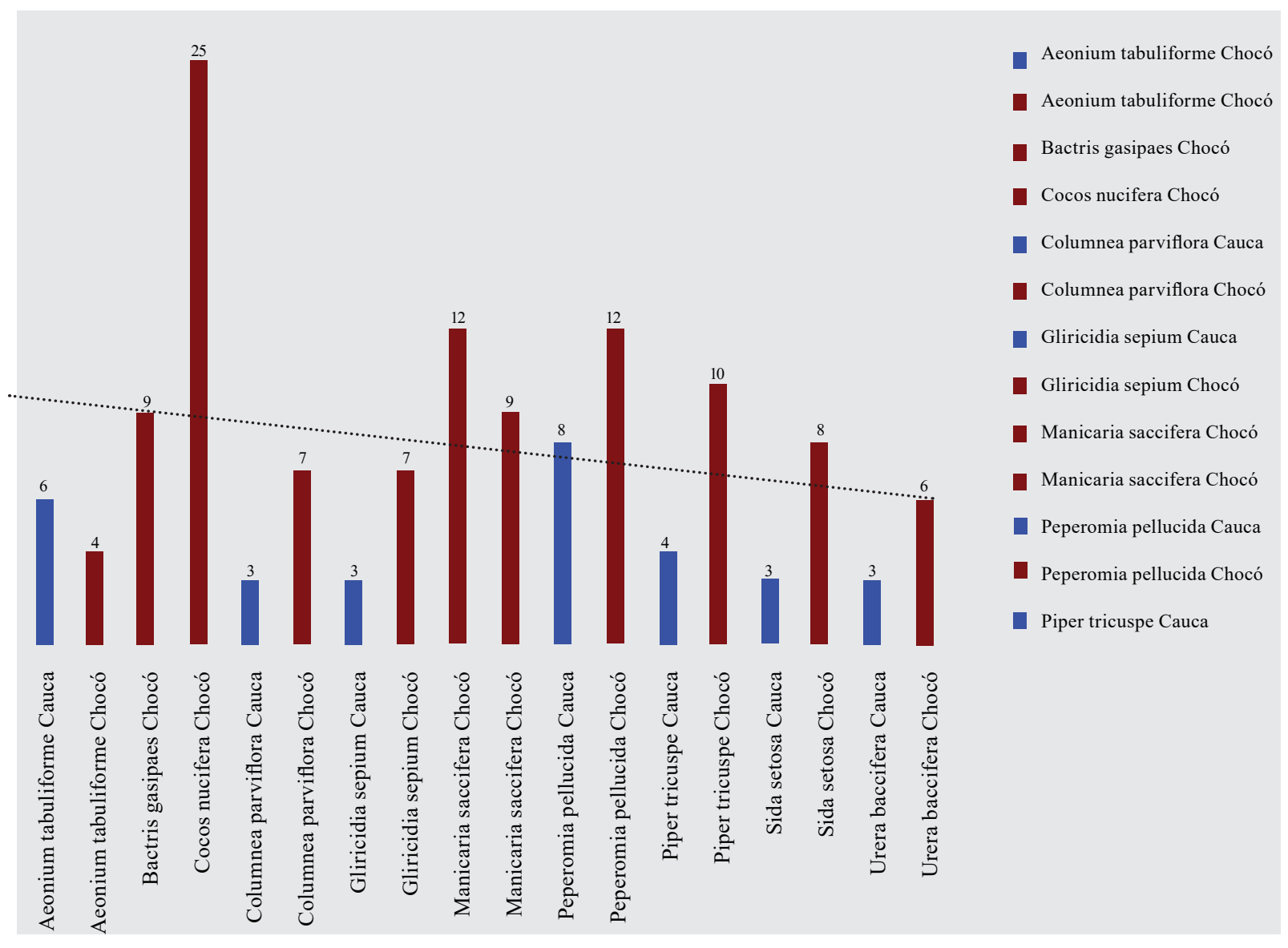

Figura 3. Comparación de especies con mayor número de usos por departamentos 


\subsection{Categorías medicinales}

Las categorías medicinales ordenadas de mayor a menor por su frecuencia de mención y número de especies vegetales que incluyen son:

\subsubsection{Contra veneno}

Las plantas medicinales incluidos en esta categoría son los utilizados para tratar mordeduras de serpientes como la equis (Bothrops spp.), la verrugosa (Lachesis muta) y la coral (Micrurus sp.). También se incluyen plantas que tratan las picaduras de rayas, hormiga como la conga (Paraponera spp.), arañas y alacranes. El 46\% (Tabla 3.). Las especies medicinales más representativas para esta categoría fueron reportadas para los afrocolombianos. En esta categoría las familias con más registros fueron, Piperaceae especialmente del género Piper. Jiménez [38] en un análisis comparativo entre el Chocó Biogeográfico y otros lugares del mundo en relación con el uso de plantas medicinales para la cura y/o alivio de las afecciones ocasionadas por la mordedura de serpientes, reporto que las especies más importante para la cura de este mal son: Piper tricuspe y Piper darienense en este mismo sentido López y Soto [39] evidenciaron 8 especie del género Piper utilizadas para la contrarrestar el veneno por mordedura de serpientes.

Tabla 3. Categorías de uso medicinal en relación al número de familias y especies

\begin{tabular}{|l|c|c}
\hline \multicolumn{1}{|c|}{ Categorías medicinales } & $\begin{array}{c}\text { Número } \\
\text { de familias }\end{array}$ & $\begin{array}{c}\text { Número } \\
\text { de especies }\end{array}$ \\
\hline Contravenenos & 103 & 297 \\
\hline Sistema digestivo & 92 & 161 \\
\hline $\begin{array}{l}\text { Síntomas sin especificary } \\
\text { znfermedades generales }\end{array}$ & 77 & 145 \\
\hline Infecciones e infestaciones & 72 & 137 \\
\hline Enfermedades culturales & 45 & 80 \\
\hline Piel y tejidos subcutáneos & 38 & 79 \\
\hline Sangre y sistema circulatorio & 24 & 50 \\
\hline Aparato reproductor & 42 & 42 \\
\hline Sistema respiratorio & 24 & 37 \\
\hline Sistema músculo-esquelético & 17 & 35 \\
\hline Sistema urinario & 13 & 32 \\
\hline Embarazo, parto y puerperio & 9 & 21 \\
\hline Salud dental & 7 & 19 \\
\hline Sistema nervioso y salud mental & 5 & 15 \\
\hline Sistema sensorial & 5 & 12 \\
\hline Sistema endocrino & 3 & 11 \\
\hline Otros & 4 & 5 \\
\hline
\end{tabular}

\subsubsection{Sistema digestivo}

Los problemas digestivos son tratados por el $26 \%$ de las especies. Las más comúnmente usadas son Ricinus communis (Igerilla) Ficus luschnathiana(Higueron) Citrus limon Limon, Se usan para tratar sobre todo afecciones como dolores de estomacales, parasitos, diarrea. Se encontraron, además, plantas que alivian intoxicaciones por ingesta de algunos alimentos Chelidonium majus (ceedonia) Las plantas utilizadas para tratar el resto de problemas digestivos afecciones son menos numerosas.

\subsubsection{Síntomas sin especificar y enfermedades generales}

Los sistemas sin especificar y enfermedades en general registra el $17 \%$ es decir, que son para aliviar las manifestaciones de enfermedades que pueden o no ser diagnosticadas por el enfermo o el tratante. Las plantas incluidas en esta categoría alivian estas manifestaciones fácilmente perceptibles, pero no curan la enfermedad que las ocasiona en sí. Las familias con más registros fueron Asteraceae, Solanaceae, Fabaceae y Lamiaceae. Dentro de esta categoría, la mayoría de las especies se usa para combatir dolores como los de cabeza, dolor del cuero. Las especies más usadas para este fin son introducidas, entre ellas la hierba luisa (Cymbopogon citratus), la ruda (Ruta graveolens) y la manzanilla (Matricaria recutita). Se registraron especies que se utilizaba para bajar la fiebre, principalmente la verbena (Verbena litoralis), la wawallpa panka pequeña (Mollinedia ovata) y la borraja (Borago officinalis). Otra que son emplean para combatir la tos y destacan para el efecto el isu (Dalea coerulea) y el poleo o tipo blanco (Minthostachys mollis).

\subsubsection{Infecciones e infestaciones}

Las plantas para tratar infecciones e infestaciones constituyen el $13 \%$ del total de especies medicinales. En esta categoría se incluyeron las especies utilizadas para tratar afecciones causadas por bacterias, virus, hongos, protozoos, platelmintos, nematodos, anélidos y artrópodos. Familias con gran número de registros fueron Asteraceae, Solanaceae y Fabaceae, se encuentran especies para curar abscesos con pus, gonorrea, herpes, sarampión, erisipela, neumonías y otras enfermedades infectocontagiosas 


\subsubsection{Enfermedades culturales}

En esta categoría están incluidas el 11\% de las especies medicinales registradas, en el tratamiento de enfermedades como el ojo, arebato, amansar niños bravos. Para cansar cangejos se ha empleado a Heteranthera reniformis y Drymonia pendula que pertenecen a las familias Pontederiaceae y Gesneriaceae, respectivamente.

\subsection{Grupos étnicos}

$\mathrm{Al}$ analizar el uso de las plantas medicinales a nivel de los grupos étnicos de Chocó y el Cauca se reflejó que el $57 \%$ de los registros medicinales corresponde a grupos de afrocolombianos, el 36\% a indígena y el $7 \%$ a grupos de mestizos (Figura 4 ) demostrando así la diversidad de saberes por parte de los diferentes grupos, por otra parte, muchos investigaciones muestran que los diferentes grupos humanos, sociales y económicos presentan patrones distintos en cuanto a la forma como perciben la salud/enfermedad y cómo actúan frente a ella. Las subculturas populares, resultado de una mezcla de diversos elementos culturales, tienen modelos conceptuales para explicar el origen de la enfermedad; estos van, desde concepciones mágico-religiosas, hasta el extremo positivista. Pasan por el espectro de variadas interpretaciones y prácticas de diagnóstico, prevención, tratamiento y rehabilitación acordes con esa cosmovisión [40].

Según Bedon [41] el estudio de las plantas relacionado a su uso por los pueblos, tanto de Afros como de indígenas y otros colonizadores. Ha sido de gran utilidad en la Atención Primaria, tanto para mejorar, prevenir, curar padecimientos y para el desarrollo de medicamentos, ya que el ser humano siempre ha utilizado las plantas para enfrentar las enfermedades [42]. Es probable que el número de especies medicinales reportadas para los indígenas sea mayor, ya que gran parte del registro no tienen datos de grupos humanos específicos. el grupo étnico que usa una mayor cantidad de especies medicinales son la de los afrocolombianos por consiguiente reflejaron usos para todas las 17 categorías de nuestra investigación.

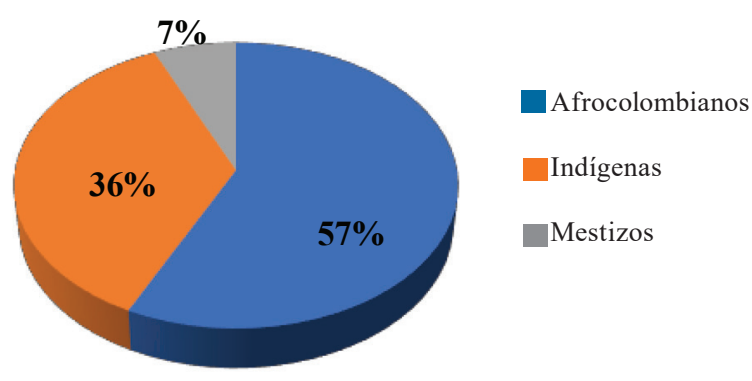

Figura 4. Usos medicinales reportados por grupos étnicos de Cauca y Chocó

\section{CONCLUSIONES}

Los resultados de esta revisión bibliográfica ponen en el relieve el papel tan importante que juegan las plantas medicinales en los departamentos de Chocó y Cauca. Esta región ostenta uno de los índices de calidad de vida más bajo de Colombia y se refleja en la situación de la salud de las comunidades, ya que tienen mayor predisposición a enfermarse, (tuberculosis, parásitos intestinales, cólera) como a la desnutrición. Esto tiene una relación directa con distintos aspectos de pobreza y una condición precaria de los grupos étnicos asentados en el territorio, que los lleva a hacer uso de las plantas medicinal para satisfacer sus necesidades medicinales básicas. Por esta razón consideramos que es muy importancia la medicina tradicional como parte fundamental de la cultura de los pueblos, la cual ha sido durante siglos, el único sistema utilizado en la restauración de la salud, donde las plantas medicinales han cumplido un rol primordial como medio para curar enfermedades en las personas. Dejando muy en claro que el sistema tradicional sigue teniendo presencia, en las comunidades de indígenas, mestizos y sobre todo en los afrocolombianos, que es donde más predominio de los saberes de plantas medicinales se han acumulado desde prácticas ancestrales de selección, manejo y conservación de conocimientos que se han transmitido de una generación a otra. Se propone la necesidad de investigaciones interdisciplinarias y participativas, para contribuir en el conocimiento de la dinámica, valoración y manejo de los diferentes ecosistemas en los estos departamentos. Es necesario, entonces, hacer esfuerzos para evitar la pérdida definitiva del conocimiento tradicional sobre plantas medicinales, no solo para preservar esta herencia cultural, sino también para registrar la información sobre ciertas especies útiles, que podrían ser relevantes para el desarrollo de nuevas fuentes de medicamentos y de otros beneficios para la humanidad, contribuyendo, al mismo tiempo, a proteger la biodiversidad.

\section{BIBLIOGRAFÍA}

[1] Organización Mundial de la Salud. 2019. https://www.who.int/healthtopics/traditio nal-complementary-and-integrative-medi cine\#tab=tab 1 
[2] C. Rentería, \& M.A. García. Evaluación de la efectividad de una composición etonofarmacológica dirigida a la cura y/o alivio de la gastritis. Bioetnia 8(1): 94-104, 2011.

[3] P. Shrestha \& S. Dhillion. Medicinal plant diversity and use in the highlands of Dolakha district, Nepal. J. Ethnopharmacol. 86: 81-96, 2003.

[4] S. Katewa, B. Chaudhary \& A. Jain Folk herbal medicines from tribal area of Rajastan, India. J. Ethnopharmacol. 92: 41-46, 2004.

[5] E. Rengifo, S. Rios, L. Fachín \& G. Vargas. Saberes ancestrales sobre el uso de flora y fauna en la comunidad indígena Tikuna de Cushillo Cocha, zona fronteriza Perú-Colombia-Brasil. Revista peruana de biología. I (24), 2017.

[6] G. Nigenda, A. Mora \& E. Orozco. La práctica de la medicina tradicional en América Latina y el Caribe: el dilema entre regu- lación y tolerancia. Salud Pública de México.43(1), 2001.

[7] G. Shoshana, E. Ito, A. Vargas. Enfermedades y padeceres por los que se recurre a te- rapeutas tradicionales de la Ciudad de Mé- xico. Salud pública de México. 48(1): p. 45-55, 2006.

[8] A. Villanueva. Identidad social y paisaje cultural en la comunidad indígena Embera Chamí de la vereda San Cayetano del municipio de Supía, Caldas, Colombia. NOVUM, l(10), 100-123, (2020).

[9] Royal Botanic Gardens, Kew. The State of the Worlds Plants Report. 2016.

[10] A. Friedl, C. Woodcock, S. Gopal, D. Muchoney, H Strahler, \& C.Barker. A note on procedures used for accuracy assessment in land cover maps derived from AVHRR data. International Journal of Remote Sensing, 21, 1073-1077, 2010.

[11] H. Gamboa, \& Y. Mosquera. Caracterización Etnobotánica y Valor de Económico de Uso Directo de las Especies Frutales Silvestres Comestibles en el Corregimiento de Samurindó Atrato, Chocó-Colombia Tesis en Biología. Universidad tecnológica del Chocó, 2014.
[12] O. Rangel, \& O. Rivera. Diversidad y Riqueza de Espermatófitos en el Chocó Biogeográfico. En: Rangel, O (Ed), Colombia Diversidad Biótica IV, Instituto de Ciencias Naturales, Universidad Nacional de Colombia. Bogota. pp. 83-99, 2004.

[13] R. Bernal, \& G. Galeano. Las palmas del Andén Pacífico. En: P. Leyva (ed.) Colombia Pacífico. Fondo FEN Colombia- Bogotá: pp. 221-231, 2006.

[14] C. Cabrera \& J. Wilder. Metodologías de muestreo de la diversidad florística. 2020

[15] Rangel \& Rivera. Colombia diversidad biótica IV. El Chocó Biogeográfico/Costa Pacífica. Universidad Nacional de Colombia. Bogotá, D.C. 2004

[16] DANEhttps://www.dane.gov.co/index. php/estadisticas-por-tema/demografia-ypoblacion/censo-general-2005-1

[17] J. Cook \& D. Sackett. The number needed to treat: a clinically useful measure of treatment effect. Bmj, 310(6977), 452-454, 1995.

[18] M. Macía, P. Armesilla P., R. Camra - S. Zambrano, H. Villalba \& M Pardo. Palma uses in North- westerne South America: A Quantitative Review.Botanilcal. Review.77 (4): 462- 560, 2011.

[19] M. Gruca, R. Cámara, M. Macía, \& H. Balslev. New categories for traditional medicine in the Econ. Bot. Data Collection Standard. J Ethnopharmacol 155: 1388 -1392, 2014.

[20] L. De la Torre, H. Navarrete, P. Muriel, M. Macía \& H. Balslev. Enciclopedia de las plantas útiles de Ecuador. Pontificia Universidad Católica Ecuador/Universidad de Aarhus, Quito. 2008.

[21] E. Ledezma. Etnobotánica de las palmas en las tierras bajas del Pacífico colombiano, con énfasis en la palma cabecinegro (Manicaria saccifera Gaertn). Departamento de Biología, 2011.

[22] A. Gutiérrez \& R. Peralta. Palmas comunes de Pando. Proyecto de Manejo Forestal Sostenible de Pando (PANDFOR),2001.

[23] M. Escalante \& P. Martínez. Sensibilidad de Listeria monocytogenes y Listeria ivanovii frente al aceite esencial de Cocos nucifera. REBIOL, 36(1), 38-44, 2020. 
[24] E. Rentería, J. Copete, L. Núñez, I. Olivares \& G. Galeano. Biología poblacional y reproductiva, usos y manejo de la palma cabecinegro (Manicaria saccifera) en los bosques inundables del Chocó, Colombia. XIV. morichales, cananguchales y otros palmares inundables de suramérica, 407, 214.

[25] Y. Olivero, D. Cano Valido, J. Guzmán, Y. Oquendo, \& N. Cruzata, Efecto diurético de Peperomia pellucida en ratas Wistar. Revista Información Científica, 62(2), 2009.

[26] N. Benítez. Actividad antibacteriana a partir de extractos de hojas de seis especies del género Piper L. (Piperaceae). Revista Institucional Universidad Tecnológica del Chocó, 27(1), 67-75, 2008.

[27] M. Rojas, G. Garavito \& J. Rincón. Actividad antiplasmódica in vitro de Piper holtonii (cordoncillo). Revista Cubana de Plantas Medicinales, 19(1), 69-75. 2014.

[28] C. Veloz, D. Altagracia, \& E. Figueroa. Validación de los efectos terapéuticos por el uso de la Peperomia pellucida L. sobre diferentes afecciones de salud en los municipios Sabana Grande de Boyá y Bayaguana, Provincia Monte Plata, Región Este de la República Dominicana,2019.

[29] N. Benítez \& E. Stashenko. Validación antibiótica de plantas medicinales noroeste de Colombia contra Staphylococcus aureus. Boletín Latinoamericano y del Caribe de plantas medicinales y aromáticas, $8(2), 145$ 150, 2009.

[30] M. Ramos, S Ramos \& R. Franklin. Características farmacognósticas de las hojas de alternanthera lanceolata (benth.) schinz "lancetilla" proveniente del distrito de Urpay provincia de Sánchez Carrión región La Libertad 2016.

[31] Mesa J, Blair S, Saez J, Correa A, Car- mona J. In vitro antimalarial evaluation of extracts of the plants Alternanthera lanceolata and Pollalesta discolor. Vol. 94.P. 67, 1998.

[32] J. Quintanilla. Caracterización de compuestos fenolicos presentes en el extracto etanolico de hojas de Alternanthera lanceolata (Benth.) Schinz, Lancetilla. Revista Pe- ruana de Medicina Integrativa, vol. 2, no 3, p: 773-778, 2017.
[33] C. Ríos. Aportes etnobotánicos y aportes al conocimiento del nacedero. Memorias III Seminario Internacional Desarrollo Sostenible en Sistemas Agrarios. CIPAV. Cali, Colombia. p. 121, 1994.

[34] J. Suárez, \& M. Milera. Nacedero (Trichanthera gigantea). Pastos y forrajes, 19(3), 1996.

[35] D. Martínez M. López D. \& F. Montejo, Efecto antihiperglucémico del extracto metanólico de Hamelia patens Jacq.

[36] E. Forero \& A. Gentry. Lista anotada de las plantas del departamento del Chocó. Instituto de Ciencias Naturales, Universi- dad Nacional de Colombia, Editora Guada- lupe, Bogotá, 1989.

[37] O. Rangel, \& P. Lowy, Tipos de vegetación y rasgos fitogeográficos de la región pacífica de Colombia. En: Leyva-F., P. (Ed.). Colombia Pacífico. Tomo I. Fondo para la Protección del Medio Ambiente José Celestino Mutis, FEN Colombia, Santafé de Bogotá, 182-198, 2003.

[38] C. Jiménez. Análisis comparativo entre el Chocó Biogeográfico y otros lugares del mundo en relación con el uso de plantas medicinales para la cura y/o alivio de las afecciones ocasionadas por la mordedura de serpientes Comparative analysis between the Chocó Biogeography and elsewhere in connection with the use of medicinal plants for cure and/or relief of. Revista Bioetnia, Volumen 9 N 1 enero-junio, 2012 ISSN 1990-0561, 9(1), 73, (2012).

[39] J. López \& J. Pérez. Plantas alexitéricas: antídotos vegetales contra las picaduras de serpientes venenosas, 2012.

[40] A. Díaz, N. Cataño, H. López, \& V. Velásquez. Diversidad cultural de sanadores tradicionales afrocolombianos: preservación y conciliación de saberes. Aquichan, 11(3), 287-30, (2011).

[41] A. Bedón. Utilidad de plantas medicinales en el parto tradicional en la comunidad de Limoncocha Shushufindi, Sucumbíos (Bachelor's thesis), 2016.

[42] J. Saldaña. Historia social de las ciencias en América Latina. UNAM, 1996. 


\section{ANEXO 1.}

Trabajos consultados en el desarrollo de la revisión

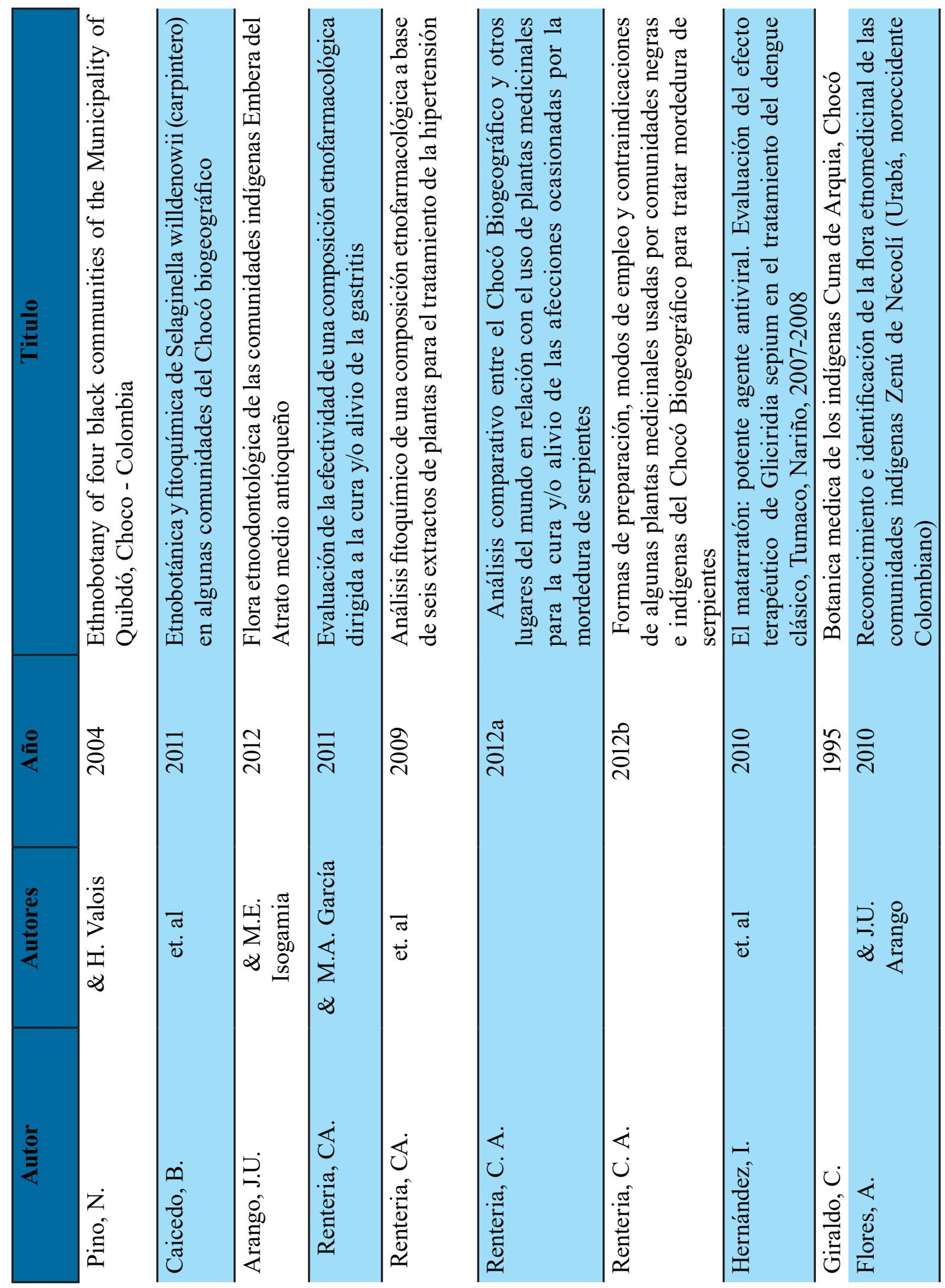

